\title{
HUMAN CHORIONIC GONADOTROPIN (HCG) STIMULANT \\ TO IMMUNOGLOBULIN FORMATION IN HUMAN \\ EMBRYONIC LYMPHOID APPARATUS BY \\ FLUORESCENT ANTIBODY TECHNIQUE
}

\author{
KANJI KASAI* AND ToshIo NISHIMURA** \\ Department of Obstetrics \& Gynecology, Faculty of Medicine, \\ Kyoto University, Kyoto
}

YosHIHIRO HAMASHIMA***

\begin{abstract}
Department of Pathology, Faculty of Medicine, Nihon University, Tokyo
\end{abstract}

Received for publication May 29, 1968

\begin{abstract}
Remarkable increase of quantity of HCG in earlier stage of normal pregnancy has been requested in further investigation on its biological and immunological properties. Normal human embryo presented the production of immunoglobulins in the medullar thymus which was the initial responsive organ among the lymphoid apparatus of human embryo in the 12th week of gestation. The so-called blood thymic barrier was apt to be broken by continuous strong stimulation of HCG to promote the histological and functional development of the medullar thymus in production of human embryonic immunoglobulins.

The detection was done by fluorescent antibody technique on normal human embryos which were obtained at legitimate induced abortion or at spontaneous abortion due to mainly incompetent cervix.
\end{abstract}

Many studies correlating the onsent of the initial immune response with immunoglobulin formation have been reported ontogenically in human embryo. The synthesis of immunoglobulins in human embryonic lymphoid apparatus has been reported by means of fluorescent antibody technique or by other effective methods $(2,5,17)$. Much has been discussed on the problem from what sort of lymphatic apparatus or lymphoid tissues, the initial immune response will be brought out in human embryo. Hanaoka (6) reported that lamina propria of G.I. series of human embryo, there could be detected no distribution of antibody formation cells throughout the course of gestation. In spite of this, Furth (2) and Mellors (11) reported the production of immunoglobulins in human embryo. There is a report describing that embryonic immunoglobulins were mostly transfered from

* The Japan Baptist Hospital, 47-Yamanomoto-cho, Kitashirakawa, Sakyo-ku, Kyoto.

** Professor and Chairman, Kyoto University, Kawaramachi, Shogoin, Sakyo-ku, Kyoto. *** Professor, Nihon University, Oyaguchi, Itabashi-ku Tokyo. 
maternal side (18) via placenta, via amnion and via amniotic fluid. Silverstein (16) indicated that the development of lymphatic apparatus, in connection with immunoglobulin production, was brought up with two different kinds of stimulation; one was specific to immunologic development in proliferating the antibody formation cells to produce antibodies, and the other was non-specific one which promoted the development of immature lymphatic apparatus to produce IgG. Auerbach (1) and Miller (12) observed that thymus had its own specific functional attitude to immune response in embryonic stage which was exceptional to any other lymphatic apparatus.

Pregnancy either intra-uterine or ectopic is, in a sense, a mechanism to establish a homologous auto-implantation in aseptic surroundings conspicuously in connection with remarkably augumented human chorionic gonadotropin (HCG) in both serum and urine of the pregnant. It is well known fact that there is neither equilibrium nor parallelism between histological and functional development of placenta and the quantitative increase of HCG with either biological or immunological method of assay against HCG.

The authors have been much interested in the problems of immunological influences of HCG on intra-uterine development of human embryo in relation to the implantation of fertilized egg into endometrium and its subsequent immunologic reactions histologically and functionally between them.

\section{MATERIALS AND METHODS}

\section{Antigens and Immunizations :}

a. Human chorionic gonadotropin (HCG): HCG was extracted from urine of normal pregnancies in their first trimester. It was purified according to lyophilization to $8.4 \times 10^{3}$ I.U. per $\mathrm{mg}$ of the biological activity. Five $\mathrm{mg}$ of HCG were used for immunization primarily. The protein in HCG was precipitated with $10.0 \%$ alum solution at $\mathrm{pH} 6.40$, adjusted with $5 \mathrm{~N} \mathrm{NaOH}$ solution. It was spun at 2500r.p.m. for $20 \mathrm{~min}$ at $4^{\circ} \mathrm{C}$. The precipitate was washed with isotonic saline for several times of change and finally was dissolved in $1.0 \mathrm{ml}$ of isotonic saline. This was injected into a male albino-rabbit weighing $3 \mathrm{~kg}$ subcutaneously. Subsequently, in the 6th, 8th and 10 th week, $3 \mathrm{mg}$ of the same HCG were injected intravenously as the booster shots. Ouchterlony's agar diffusion technique and precipitin method between antigen and its specific antibody reaction in vitro revealed sufficient elevation of antibody titer in the animal.

b. Immunoglobulin $\mathbf{G}(\mathbf{I g G})$ : IgG was obtained from pooled normal human sera. From them, crude globulins were separated by repeated precipitation with $40 \%$ ammonium sulfate and then dissolved in isotonic saline. It was brought to dialysis against $0.01 \mathrm{M}$, pH 7.0 buffered saline to wash off the ammonium sulfate. These crude globulins were applied to the DEAE cellulose column chromatography in $0.01 M$ phosphate buffer at $\mathrm{pH} 8.0$ and eluted the first peak being IgG globulin. The $\mathrm{pH}$ of the eluted buffer remained constant. This chromatographic procedure was carried out at room temperature to prevent denaturation in the cold. Immunoelectrophoretic analysis revealed the purity of $\operatorname{IgG}$ with one distinct band in fast 
gamma region. Ten $\mathrm{mg}$ of $\mathrm{IgG}$ were injected into a mature albino-rabbit subcutaneously after sufficient mixing with Freund's adjuvant. In the 5th, 7 th and 10 th week after the primary immunization, three times of booster shots were done intravenously with $4 \mathrm{mg}$ of IgG. Satisfactory elevation of the antibody titer was recognized in the animal by agar diffusion technique. Immunoelectrophoretic analysis proved the purity of the antibody against antigen IgG. In order to attain maximal capacity, the DEAE cellulose was prewashed with $1 N \mathrm{NaOH}$ and $1 N \mathrm{HCl}$ followed by distilled water. Then it was successively washed with eluting buffer until stable $\mathrm{pH}$ was obtained.

c. IgA : IgA was collected from pooled human colostrum. It was separated by gradient eluting system of phosphate buffer solution from $0.001 M, \mathrm{pH}$ 8.0 to $0.5 M$, pH 6.0 and applied to the DEAE cellulose column chromatography. The eluted IgA solution was concentrated by $20 \%$ polyvinylpyrrolidone to the content of $4 \mathrm{mg}$ of $\operatorname{IgA}$ per $\mathrm{ml}$ and then dialyzed against $0.01 M$ phosphate buffer at $\mathrm{pH}$ 7.2. This pure IgA solution showed only one band distinctly in slow gamma region immunoelectrophoretically. Ten mg of Ig A were mixed with Freund's adjuvant to be water in oil. It was injected into a mature albino-rabbit subcutaneousy to immunize the animal. In the 4 th, 7 th and 10th weeks after the primary immunization, booster shots were done intravenously with $4 \mathrm{mg}$ of $\operatorname{IgA}$ at each trial. The elevation of the anti-IgA antibody titer was sufficiently recognized in the animal by agar diffusion technique, the purity of antibody was proved by immunoelectrophoretic analysis.

d. IgM : IgM was obtained from the serum of IgM-macroglobulinemia. Ammonium sulfate of $40 \%$ saturation was applied to remove the albumin fractions. The crude globulins were precipitated and dissolved subsequently in isotonic saline to dialyze against phosphate buffer saline at pH 7.0. The gamma globulin fraction was collected from the first peak at the DEAE cellulose column chromatography, which was eluted by $0.01 M$ phosphate buffer at pH 8.0. It was rechromatographed on Sephadex G-200 column with an elute of $0.1 \mathrm{M}$ Tris $-\mathrm{HCl}$ buffer at $\mathrm{pH} 8.0$ with $0.5 \mathrm{M} \mathrm{NaCl}$. All these procedures were carried out at $4^{\circ} \mathrm{C}$.

The eluted IgM solution was concentrated to the content of $3 \mathrm{mg}$ of IgM per ml with $20 \%$ polyvinylpyrrolidone. IgM was detected in slow gamma region without any contamination immunoelectrophoretically. Twelve $\mathrm{mg}$ of this pure $\operatorname{IgM}$ were mixed with Freund's adjuvant and injected subcutaneously into a male albino-rabbit weighing $3 \mathrm{~kg}$ successively. In the 4 th, 7 th and 10 th week after the primary immunization, booster shots were done intravenously with $5 \mathrm{mg}$ of the antigen $\operatorname{IgM}$ at each trial. The elevation of the antibody titer against antigen IgM was satisfactory to take out the antiserum from the animal.

\section{Fluorescent Dye Conjugation to Antisera:}

Anti-HCG-, anti-IgG-, anti-IgA- and anti-IgM-antisera were taken out from the animals by heart puncture. Crude globulins from each of these antisera were prepared by four successive precipitations with $40 \%$ ammo- 
nium sulfate. They were dissolved separately in isotonic saline to bring the final protein concentration between 10 and $20 \mathrm{mg}$ per $\mathrm{ml}$. The solutions were dialyzed in buffered saline at $4^{\circ} \mathrm{C}$ for 5 days by frequent changes of the buffered saline until the dialyzing buffer being free from ammonium. The protein concentration was prepared to $10 \mathrm{mg}$ per $\mathrm{ml}$ and $\mathrm{pH}$ was adjusted to 9.2 by adding $0.5 \mathrm{M}$ sodium carbonic buffer. Fluorescein isothiocyanate (FITC)* or Tetra methylrhodamin isothiocyanate (TRITC)* was mixed with proper antibody at $4^{\circ} \mathrm{C}$ for $6 \mathrm{hr}$ (FITC) and $18 \mathrm{hr}$ (TRITC) with continuous stirring. The mixtures were successively dialyzed in buffered saline at $\mathrm{pH}$ 7.2. The fluoresecnt dye-protein ratios of the conjugates were prepared to be between 1 and 2 . Each immunoglobulin conjugate was treated by alternated absorption before use; anti-IgG and anti-IgA, anti-IgG and anti-IgM, anti-IgA and anti-IgM were combined for mutual absorption.

\section{Preparation of Tissue Sections and Fluorescent Staining Procedures:}

Spleens, livers, thymuses, kidneys, inguinal lymphnodes and bone marrows were collected from normal human embryos weekly from the 7 th to the 28 th of normal human gestation at legitimate induced abortion. In cases of later than the 29 th week, materials were obtained at spontaneous abortion due to mainly incompetent cervix. Paperelectrophoresis and immunoelectrophoresis on maternal sera presented nothing of blood diseases. Embryonic organs showed normal development coincidently with gestational week histologically.

The materials except bone marrows were fixed in $95.0 \%$ cold ethanol immediately after obtainance. They were kept at $4^{\circ} \mathrm{C}$ for $24 \mathrm{hr}$ and then brought into $99.0 \%$ cold ethanol. After dehydration with $100.0 \%$ cold ethanol, they were embedded in paraffin according to Sainte-Marie's procedure (14). These paraffin blocks were sectioned by microtome into $3 \mu$ thick slices, which were fixed on non-fluorescent thin glass-slides and dried at $38^{\circ} \mathrm{C}$ for $30 \mathrm{~min}$. After removal of paraffin by three changes of xylene within $15 \mathrm{~min}$, the slices were passed gently from $100.0 \%$ to $45 \%$ ethanol gradiently. The sections were then washed sufficiently in successive baths of cold buffered saline $(\mathrm{pH} 7.2)$ and wiped dry except for the areas containing the sections and then exposed to fluorescein-labelled antibody in moits chamber.

Sequential mixed staining was performed. Contrasting labelled antisera were applied consecutively The first labelled antiserum was allowed to react for one day then washed in cold buffered saline $(\mathrm{pH} 7.2)$ and the second labelled antiserum was applied overnight. Zeiss fluorescent microscope was used for examination of them.

Bone marrows were brought to stamp preparations on non-fluorescent thin glasses. They were fixed in $95.0 \%$ cold ethanol for several minutes and incubated at $38^{\circ} \mathrm{C}$ for $1 \mathrm{hr}$ in it. They were washed in buffered saline $(\mathrm{pH}$ 7.2) and brought to the staining as described above.

\footnotetext{
* Baltimore Chem. Lab.,U.S.A.
} 


\section{RESULTS}

\section{Localization and distribution of HCG in human embryo :}

HCG is known to be produced in syncytiotrophoblasts of human chorionic villi showing the maximal quantity of secretion in the 8 th to the 15 th week of normal gestation. Kasai established that HCG flows out from the production cells through the brush border membrane into the intervillous spaces of placenta and presents in small leucocytes in circulating blood in umbilical cord, although the detection of HCG in amnion or amniotic fluid was not carried out (7). And he concluded that HCG is easily transfered to embryonic organs via circulating blood in umbilical cord as a main pass-way.

In the present study, first in the 10 th week of gestation, HCG could be detected in small lymphocytes which localized in perivascular region of the medullar thymus of human embryo.

Also in plasma cells, distribution of HCG was clearly detected, which swarmed in the so-called blood thymic barrier. Among the embryonic organs materialized in this study, thymus was a limitted organ in the detection of $\mathrm{HCG}$.

In the 12 th week of normal pregnancy, HCG was distributed in thymus with IgM or IgG coincidently. Invading pattern of HCG through the barrier was demonstrated in medullar zone of thymus (Plates 1 and 2).

II. IgG :

IgG appeared at first in human thymus in normal embryo in the 12 th week and subsequently was detected in the spleen in the 18 th week or in periphery lymphnodes, represented by inguinal lymphnodes in the 20 th week of normal gestation.

IgG was detected in plasma cells dominantly either in thymus or lymphoid apparatus in human embryo. The most interesting finding was the cellular distribution of HCG and its influence on the production of IgG in the medullar thymus in the identical cells which contained HCG (Plate 3 ).

According to double staining method by fluorescent antidody technique, HCG which reacted against FITC labelled anti-HCG was clearly detected coexistently with IgG which reacted against TRITC labelled anti-IgG in some of the same plasma cells. IgG appeared at first in the perivascular plasma cells which were supposed to be influenced the strongest with antigenic substances.

\section{III. $\operatorname{Ig} \mathbf{A}$ :}

IgA which reacted against anti-human colostral $\operatorname{Ig} \mathrm{A}$, conjugated with TRITC, appeared at first in thymus of normal human embryo in the 25 th week of gestation in this study. With anti-IgA conjugate, we could detect the production of intracellular IgA in plasma cells which located in perivascular spaces or inter-lobular spaces in the medullar thymus and still more in the border region between medulla and cortex of the thymus. At this period of normal gestation, neither localization nor production of $\operatorname{Ig} \mathrm{A}$ was detected in any other lymphoid apparatus of human embryo. In the 


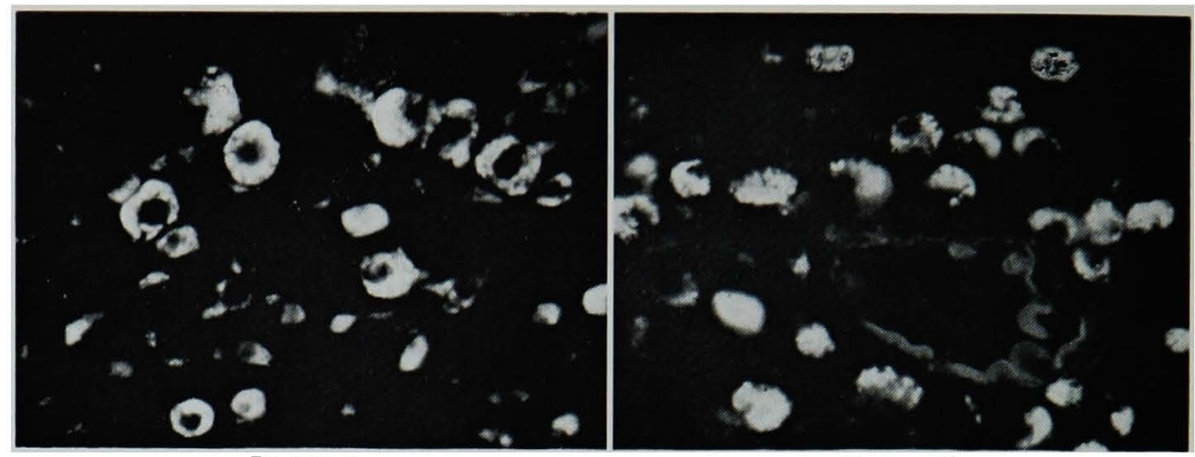

Plate 1

Plate 2
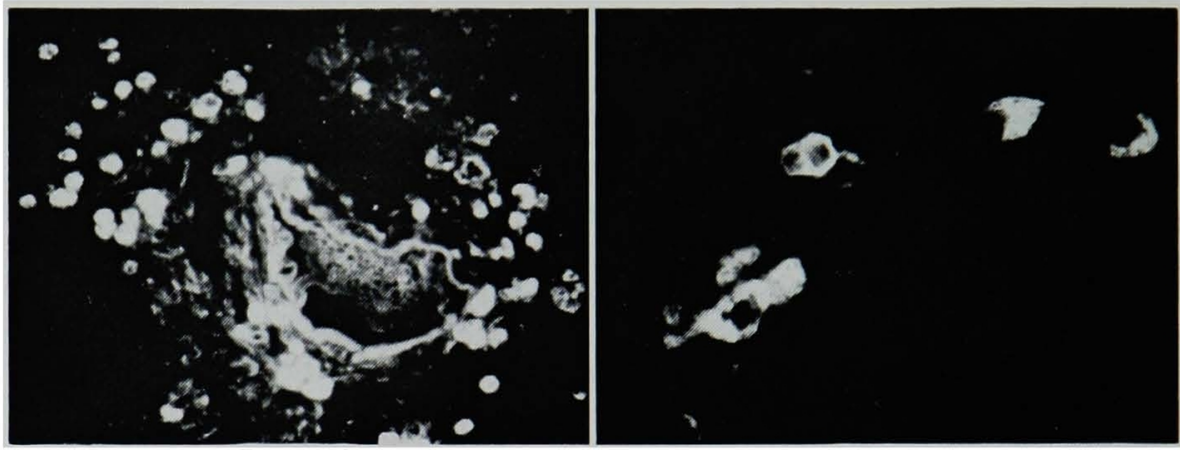

Plate 3

Plate 4

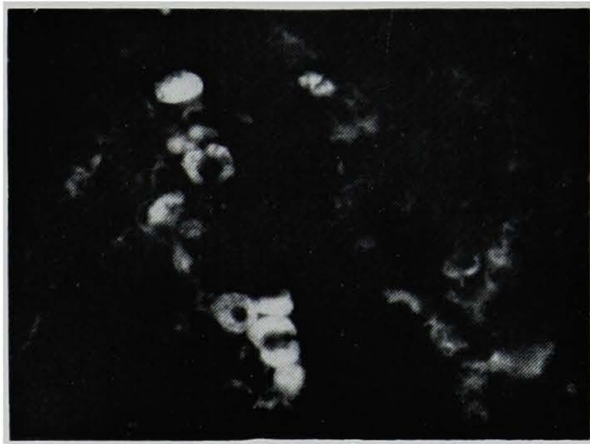

Plate 5

Plate 1. Photo shows the distribution of HCG mainly in perivascular region in the medullar thymus of normal human embryo in the 10 th week of gestation. HCG-containing cells were arranged concentrically.

Plate 2. The distribution of HCG in parenchymal cells of the medullar thymus of normal human embryo in the 14 th week of gestation. The destruction and swelling of the vascular wall were clearly demonstrated.

Plate 3. IgG originated from embryo, was detected in the medullar zone of human embryonic thymus in the 12 th week of normal gestation. Plasma cells presented the main role in the production of IgG.

Plate 4. Photo shows the distribution of embryonic IgA in the interlobular spaces of the medullar thymus in the 25 th week of normal human embryo.

Plate 5. The detection of embryonic $\operatorname{IgM}$ in the corteco-medullar thymus of normal human embryo in the 12 th week of gestation. 
spleen, IgA appeared in later than the embryo of the 32 nd week of gestation. It was here also recognized that IgA localized coincidently and coexistently in the cells in the medullar thymus which contained HCG (Plate 4).

\section{IgM :}

IgM was detected in small lymphocytes which located in perivascular region in the medullar thymus and in the border region of the cortecomedullar thymus, and appeared early in the 12 th week of normal human gestation. At the period of gestation when IgM could be detected in embryonic thymus, spleen and other lymphoid tissues were not supported histologically their functions against immune responses, i. e. they were still immature in immune responses. The onset of functional change against immunoglobulin formation was induced at first in the medullar thymus of normal human embryo with the appearance of IgM.

There was no remarkable interval of the time in the appearance of IgM and IgG with the detection by fluorescent antibody technique. As the stage of pregnancy advanced, the IgM-producing small lymphocytes increased their numbers in the medullar thymus. This phenomenon continued until the end of the 5 th month (20 th week) of gestation, which suggested that the function of thymus to produce IgM seemed to be maximized at this period of normal human pregnancy (Plate 5).

The relatonship between HCG and IgM production has required further investigation. IgM in small lymphocytes and plasma cells were recognized on the same section of the 12 th week embryo.

\section{DISCUSSION}

Whether normal human embryo can synthesize its own immunoglobulins in lymphoid apparatus in intra-uterine aseptic surroundings and if it be possible, what is the initial stimulation against immunoglobulin formation in embryo are the focalized problems in this study.

In recent years, it has been dominantly stated that thymus had no function to produce antibodies. Marshall (9) reported a hypothetic localization of blood thymic barrier between circulating blood and thymus parenchym which was supposed to interrupt the penetration of circulating antigens into thymus. But secondary nodules are reduced in the thymus of guinea-pig mainly in the inter-lobular tissues, at the corteco-medullar border and also in perivascular regions in the medullar zone. With direct, strong stimulation enough to break the barrier, thymus is said to obtain the histol. ogical change to form the secondary nodules and subsequently the increase of lymphocytes or reticular cell count as a result of antigen invation. Kotani (8) demonstrated that the barrier was not stable in protection of antigen invasion. From these facts, it may be well implied that some of antigenic substances could penetrate the barrier to stimulate the antibody formation in thymus. In this study, we could recognize the close relationship between the histological change with functional development of human embryonic thymus and the maximal increase of HCG quantitatively throughout the 
course of normal pregnancy. Previously, we could detect and reported the localization of HCG in circulating veins in umbilical cord (13) but the fate of HCG after transported into embryo had been left unclarified. In this study, FITC conjugated anti-HCG rabbit antiserum which was enough absorbed with normal human serum and successively with normal adult urine components before use, presented clearly the distribution of HCG in normal human embryo. HCG appeared at first in thymus, especially in the medullar zone, early in the 10 th week of normal pregnancy. HCG was detected in perivascular along the so-called blood thymic barrier. Sainte-Marie (15) reported that antigenic substances were taken into the medullar zone via either vessels located inter-lobular connective tissues or so-called perivascular channel in the medulla. HCG in either lymphocytes or plasma cells in embryonic thymus is closely related to the appearance of immunoglobulins.

No positive evidence has been reported on the permeability of IgM through human placenta at any period of normal gestation. As a result, it may be considered that IgM which can be detected in embryonic tissues, may be originating from embryo itself. Furth (2) reported that spleen in the 19 th week of gestation had provided the production of $\operatorname{IgM}$ of embryonic origin. Mellor and his associates (11) reported that IgM was detectable in the cells of primitive reticular appearance in embryonic thymus. In this study, IgM of embryo origin could be detected first in thymus which was prior to the detection in the spleen.

Some reports have noted that maternal immunoglobulin, especially IgG could be recognized in embryonic tissues by trans-placental pass-way (4) and as the stage of normal gestation advanced, the quantity of maternal IgG increased in embryo. Vahlquist (19) and Gitlin (3) reported that the quantity of IgG in embryo was far smaller in the 5th-6th month than that in the terminal stage of gestation. The evidences reported by Martensson and his associates (10) correlating to antibody formation in maternal serum of $\mathrm{Gm}(\mathrm{a}-)$ against $\mathrm{Gm}(\mathrm{a}+)$ fetus surely implied that IgG production could be carried out in human embryo.

Of the transference via placenta against IgA, there have been several reports in which mostly negative opinions were described. In this study, IgA was detected in neither umbilical blood nor thymus coincidently with IgG in thymus.

There is no doubt that the placental permeability against immunoglobulins depends not only upon the molecular weight and structure of gamma globulins but upon the histological changes of the placental villi including pathological degeneration of human placental tissues caused by spontaneous abortion and so on.

The distribution of HCG in either lymphocytes or plasma cells in embryonic thymus coincidently with the appearance of immunoglobulin suggests that one of main roles of HCG is to stimulate the functional development of the primitive thymus to bring out the immunoglobulin formation by inactivating against the mechanism of blood thymic barrier with continuous and quantitative stimulation. 
Further investigations are required to understand the biological and immunological properties of HCG distribution in the human embryo organs.

\section{REFERENCES}

1. Auerbach, R.: Morphogenetic interactions in the development of the mouse thymus gland. Developmental Biology 2; 271, 1960.

2. Van Furth, R. et al.: The immunological development of the human fetus. J. exp. Med. 122; 1173, 1965.

3. Gitlin, D. et al.: Serum $\alpha$-fetoprotein, albumin and $\gamma \mathrm{G}$-globulin in the human conceptus. J. Clin. Invest. 45; 1826, 1966.

4. Gouya, N. et al.: (Japanese). Shonika-Shinryo 28; 1543, 1965.

5. Hamashima, Y.: Intrauterine and neonatal infection under immature antibody formation. Acta Haemat. Jap., 30; 104, 1967.

6. Hanaoka, M.: (Japanese). The Saishin-Igaku 19; 3137, 1964.

7. Kasai, K.: The localization and distribution of HCG in villous tissues. Proceedings of the 3rd Asia and Oceania Congress of Endocrinology, Manila, Philippines, 1967.

8. Kotani, M. et al.: (Japanese). Kaibo Gakkai Zasshi 40; 23, 1965.

9. Marshall, A. H. et al.: The immunological reactivity of the thymus. J. exp. Path. $42 ; 379,1961$.

10. Martensson, L. et al.: Gm genes and $\gamma_{a}$-globulins synthesis in the human fetus. $J$. Immunol. 94; 514, 1965.

11. Mellor, R.C. et al.: The cellular origin of human immunoglobulins $\left(\gamma_{2}, \gamma_{1} \mathrm{M}, \gamma \mathrm{A}\right)$. J. exp Med. 118; 387, 1963.

12. Miller, J.F.A.P. et al.: Embryological development of the immune mechanism. Ann. Rev. Med. 15; 23, 1964.

13. Nishimura, T. et al.: (Japanese). Sanfujinka Chiryo 12; 326, 1966.

14. Sainte-Marie, G.: A paraffin embedding technique for studies employing immunofluorescence. J. Histochem. Cytochem. 10; 250, 1962.

15. Sainte-Marie, G.: Antigen penetration into the thymus. J. immunol. 91; 840, 1963.

16. Silverstein, A.M. et al.: Fetal response to antigenic stimulus. 1. plasma-cellular and lymphoid reactions in the human fetus to intrauterine infection. Lab. Invest. 11; 918, 1962.

17. Silverstein, A.M.: Ontogeny of the immune response, the development of immunologic response by the fetus. Science $144 ; 1423,1964$.

18. Tanaka, M. et al.: (Japanese). Nippon Shinseiji Gakkai Zasshi 1; 43, 1965.

19. Vahlquist, B.: The transfer of antibodies from mother to offspring. Advances Pediat. 10; 325, 1958. 